\title{
Paraplegia
}

\section{Traumatic Cervical Brown-Sequard and Brown-Sequard-plus Syndromes: The Spectrum of Presentations and Outcomes}

\author{
E. J. Roth, MD, ${ }^{1,2,}{ }^{3}$ T. Park, MD, ${ }^{2}$ T. Pang, $M D,{ }^{2}$ G. M. Yarkony, MD,,${ }^{1,2,3}$ \\ M. Y. Lee, $M D,{ }^{1,}, 2,3$ \\ ${ }^{1}$ Midwest Regional Spinal Cord Injury Care System, ${ }^{2}$ Department of Rehabilitation \\ Medicine, Northwestern University Medical School, ${ }^{3}$ Rehabilitation Institute of \\ Chicago, Chicago, Illinosis, USA
}

\begin{abstract}
Summary
Brown-Sequard syndrome (BSS) and Brown-Sequard-plus syndrome (BSPS) are characterised by asymmetrical paresis with hypalgesia more marked on the less paretic side. This study examined the clinical features of 38 patients (30 males and 8 females; mean age $=32$ years) with traumatic cervical BSS or BSPS who underwent comprehensive inpatient rehabilitation. Twenty two injuries were caused by road traffic accidents, 8 by penetrating injuries, 5 by diving injuries, and 3 by other causes. After an average of 35 days in acute care and 79 days in rehabilitation, 37 patients had increased muscle strength, all 38 patients improved functional abilities, 29 patients walked independently, 34 had spontaneous bladder emptying, 36 were discharged home, and 14 were employed. Statistically significant increases $(p<0.001)$ were made in modified Barthel index functional scores between admission and discharge. Patients with BSPS had a better prognosis than did those with 'pure' BSS. Patients with predominant upper limb weakness had more favourable outcomes than did those with predominant lower limb weakness. Few other potentially predictive demographic, injury, or neurological factors were associated with functional outcome. Patients with BSS or BSPS generally have a good prognosis for neurological and functional improvement.
\end{abstract}

Key words: Spinal cord injury; Brown-Sequard syndrome; Tetraplegia; Rehabilitation.

Although the first description of 'spinal hemiplegia' was published by Dundas in 1825 , initial characterisation of the classic syndrome of spinal hemisection causing ipsilateral hemiplegia and contralateral hemianalgesia is usually credited to Brown-Sequard in 1846. Subsequent authors (Bohlman, 1979; Bosch et al., 1971;

This work was supported in part by the U.S. Department of Education National Institute of Disability and Rehabilitation Research grant G008535129, the Midwest Regional Spinal Cord Injury Care System.

This study has been presented at the 16th Annual Scientific Meeting of the American Spinal Injury Association, May 5, 1990, Orlando, Florida, USA.

Correspondence to: E. J. Roth, MD, Rehabilitation Institute of Chicago, 345 E. Superior St., Chicago, Illinois 60611 USA. 
Braakman and Penning, 1976; Cushing, 1898; Cabezudo et al., 1980; Gentleman and Harrington, 1984; Holmes, 1915; Koehler and Endtz, 1986; Lipschitz and Block, 1962; Little and Halar, 1985; Peacock et al., 1977; Preston, 1896; Rand and Patterson, 1929; Taylor and Gleave, 1957) have documented pathological, aetiological, and clinical features in patients with this syndrome. However, only a limited number of patients have the 'pure' form of Brown-Sequard syndrome (BSS). Much more common is the constellation of findings described by Taylor and Gleave (1957) and by Braakman and Pennington (1976); 'Brown-Sequard-plus syndrome (BSPS) consists of asymmetric paresis with hypalgesia more marked on the less paretic side. Exact estimates of the frequency with which this condition occurs are difficult to determine, but syndromes of the 'Brown-Sequard' type probably account for about $2-4 \%$ of all traumatic spinal cord injuries (Bohlman, 1979; Bosch et al., 1971).

The prognosis of functional motor recovery following the onset of these syndromes is generally considered good (Bosch et al., 1971; Little and Halar, 1985; Taylor and Gleave, 1957). However, detailed empirical clinical data describing neurological, medical, and rehabilitation outcomes of patients with traumatic cervical BSS or BSPS are sparse. In order to evaluate more fully the clinical and prognostic features of this syndrome, demographic, neurological, and functional characteristics of 38 patients with traumatic cervical BSS or BSPS who underwent comprehensive inpatient rehabilitation were examined and their course of recovery was studied.

\section{Methods}

\section{Patients}

The study sample consisted of 38 patients who sustained spinal cord trauma resulting in asymmetric weakness with sensory deficit more marked on the less paretic side, who completed inpatient rehabilitation, and who underwent follow-up for at least 6 months after discharge.

\section{Procedures}

The complete medical records of all patients were reviewed. Demographic data included age, gender, race, educational level, marital status, and employment status at injury. Injury data included aetiology of injury, frequency and types of associated injuries, presence of cervical spine fracture, spinal stabilisation method, and duration between injury and rehabilitation admission.

Neurological data were determined by a review of admission and discharge manual muscle test results. Rehabilitation and follow-up data included length of rehabilitation stay, discharge location, follow-up employment status, occurrence and types of complications, methods of urinary tract management, and method of bowel regulation.

Functional data at rehabilitation admission and discharge were assessed using the Modified Barthel Index (Granger et al., 1979; Mahoney and Barthel, 1965; Yarkony et al., 1987). 


\section{Data analysis}

Descriptive statistics were used to characterise the study sample. Student t-tests were used to compare admission and discharge MBI scores and subscores. Chisquare analysis and Fisher's Exact Test were used to compare admission and discharge frequencies of independence in the performance of each of the 14 functional tasks on the MBI.

Nine specific outcomes_length of rehabilitation stay, occurrence of medical complications during rehabilitation, discharge location, employment status at follow-up, discharge bladder continence status, discharge ambulatory status, discharge MBI self-care subscore, discharge MBI mobility subscore, and discharge MBI total score-were compared between patients with pure BSS and those with BSPS using Chi-square analysis, Fisher's exact test, or Student t-tests.

Relationships were evaluated between each of 14 independent variables and each of the 9 dependent outcome measures using standard statistical methods.

\section{Results}

\section{Demographic characteristics}

The mean ( \pm s.d.) age of the 38 subjects was $32 \cdot 1 \pm 14 \cdot 1$ years, with a range between 15 and 73 years. There were $30(79 \%)$ males. Twenty five $(66 \%)$ patients were white, $10(26 \%)$ were black, and $3(8 \%)$ were Hispanic. The mean educational level was $12 \cdot 0 \pm 2 \cdot 6$ years, with a range between 8 and 20 years. At the time of the injury, $26(68 \%)$ patients were employed, $6(16 \%)$ were students, and the remainder were unemployed or retired. Nineteen $(50 \%)$ were single, $15(39 \%)$ were married, 3 (8\%) were divorced, and 1 was widowed.

\section{Injury characteristics}

SCIs were caused by road traffic accidents in $22(58 \%)$, penetrating wounds in 8 (21\%; 6 bullet and 2 stab wounds), diving in $5(13 \%)$, and other causes in 3 . Twenty nine $(76 \%)$ patients sustained cervical vertebral fractures; 4 also had thoracic or lumbar fractures. Fif teen (39\%) sustained a concurrent closed head injury, with loss of consciousness and/or post-traumatic amnesia at the time of the SCI. Seventeen (45\%) had other associated injuries; these included extremity fracture (4), skin laceration (4), hemopneumothorax (3), hemidiaphragm paralysis (3), rib fracture (2), liver injury (1), and cardiac arrest (1).

The mean duration between injury and rehabilitation was $34.9 \pm 28.6$ days, with a range between 6 and 145 days. Twenty one patients underwent surgical stabilisation of cervical spine fractures. Twenty nine medical complications occurred during the acute hospitalisation in 22 (58\%) patients; the most common were urinary tract infections, pneumonias, other infections, and dysesthetic pain.

\section{Neurological characteristics}

All patients were admitted to and discharged from rehabilitation with incomplete cervical SCIs. Five had pure BSS (caused by road traffic accident in 2, gunshot wound in 2, and diving in 1 . The remaining 33 patients had BSPS. Admission 
Frankel Grades were B in 1 and D in 37; discharge Frankel Grades were D in all 38 patients. Neurological levels were C3 in 1 patient, C4 in 19, C5 in 5, C6 in 5, C7 in 5 , and $\mathrm{C} 8$ in 3. The predominant side of weakness was right in 18 and left in the remainder. Weakness was predominant in the upper extremity in 20 patients, and in the lower extremity in 18. Neurologic improvement was noted during the rehabilitation hospitalisation in 37 patients.

\section{Rehabilitation and follow-up characteristics}

The mean length of rehabilitation stay was $79 \cdot 3 \pm 46 \cdot 0$ days (range $21-213$ days). Following rehabilitation, 36 patients went home; at follow-up, 37 (97\%) were living at home. Fourteen (37\%) were working or in school at follow-up.

During rehabilitation, 29 patients experienced 44 medical complications; the most common were urinary tract infection and dysesthetic pain. During follow-up, 13 patients experienced 17 significant complications; the most common were severe spasticity and dysesthetic pain.

Bowel and bladder status on admission and discharge is shown in the Table. At discharge, no patients required an indwelling urethral catheter or had bowel incontinence.

\section{Functional characteristics}

Statistically significant $(p<0 \cdot 001)$ improvements were made during rehabilitation in mean modified Barthel index (MBI), self-care subscores, mobility subscores, and total scores (Fig. 1). In addition, statistically significant increases $(p<0.0001)$ were noted between admission and discharge in the proportions of patients who were able to complete independently each of the 8 self-care tasks and the 6 mobility tasks of the MBI (Fig. 2 and 3). At discharge, 18 (47\%) patients were able to perform all 14 of the MBI tasks independently at discharge.

\section{Comparison between BSS and BSPS patients}

Compared to the 5 patients with 'pure' BSS, the 33 patients with BSPS had significantly shorter lengths of rehabilitation stay $(68 \cdot 9 \pm 39 \cdot 9$ vs. $142 \cdot 4 \pm 46 \cdot 8$ days;

Table Admission and discharge bladder and bowel function of 38 BSS and BSPS patients

\begin{tabular}{lcc}
\hline Bladder function & Admission & Discharge \\
Continent spontaneous & $13(34 \%)$ & $34(89 \%)$ \\
External collecting device & $3(8 \%)$ & $2(5 \%)$ \\
Intermittent catheterisation & $11(29 \%)$ & $2(5 \%)$ \\
Indwelling catheter & $11(29 \%)$ & 0 \\
& & \\
Bowel function & Admission & Discharge \\
Continent spontaneous & $5(13 \%)$ & $25(66 \%)$ \\
Suppository programme & $27(71 \%)$ & $12(32 \%)$ \\
Incontinence & $5(13 \%)$ & 0 \\
Colostomy & $1(3 \%)$ & $1(3 \%)$ \\
\hline
\end{tabular}




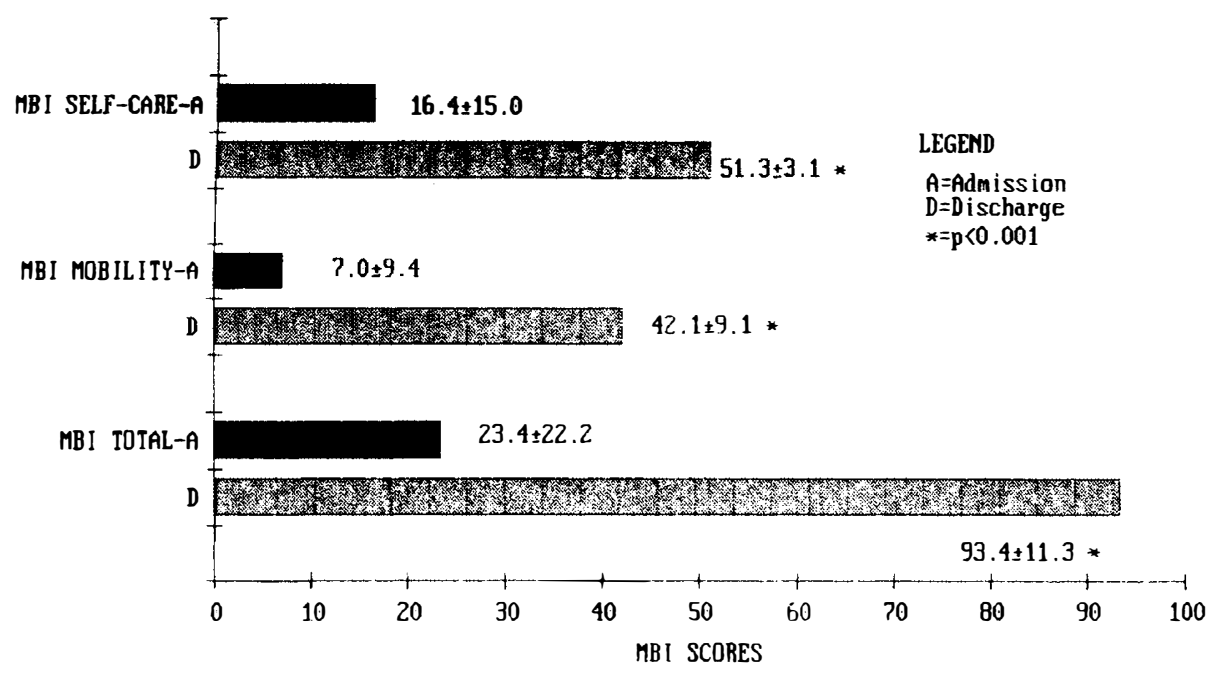

Figure 1 Modified barthel index scores at rehabilitation admission and discharge.

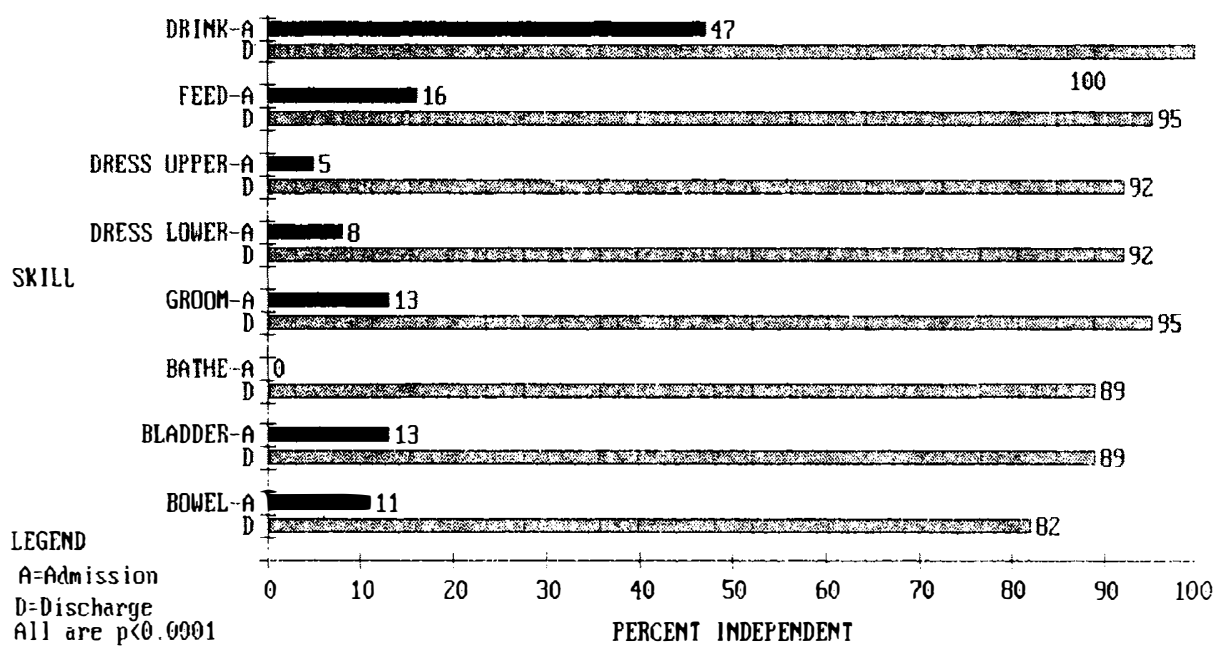

Figure 2 Proportions of patients who were independent in 8 self-care skills.

$\mathrm{t}=3.76) \mathrm{p}<0.0001)$, a greater frequency of independent ambulation $(85 \%$ vs. $20 \%$; $\left.\mathrm{X}^{2}=6.83 ; p<0.01\right)$, a greater mean MBI self-care subscore $(51 \cdot 9 \pm 2 \cdot 8$ vs. $47 \cdot 2 \pm 2 \cdot 2 ; \mathrm{t}=3 \cdot 58 ; p<0 \cdot 001)$, a greater mean MBI mobility subscore $(44 \cdot 6 \pm 5 \cdot 7 \mathrm{vs.}$ $26 \cdot 0 \pm 12 \cdot 6 ; t=5 \cdot 67 ; p<0 \cdot 0001)$, and a greater mean MBI total score $(96 \cdot 5 \pm 7 \cdot 2$ vs. $73 \cdot 2 \pm 14 \cdot 2 ; \mathrm{t}=5 \cdot 87 ; p<0 \cdot 0001)$. There were no differences between BSS and BSPS groups with respect to complication frequency, discharge bladder status, discharge location, or follow-up employment status.

\section{Predictors of outcome characteristics}

Only 6 of 126 possible associations between the 14 potential predictor variables and 


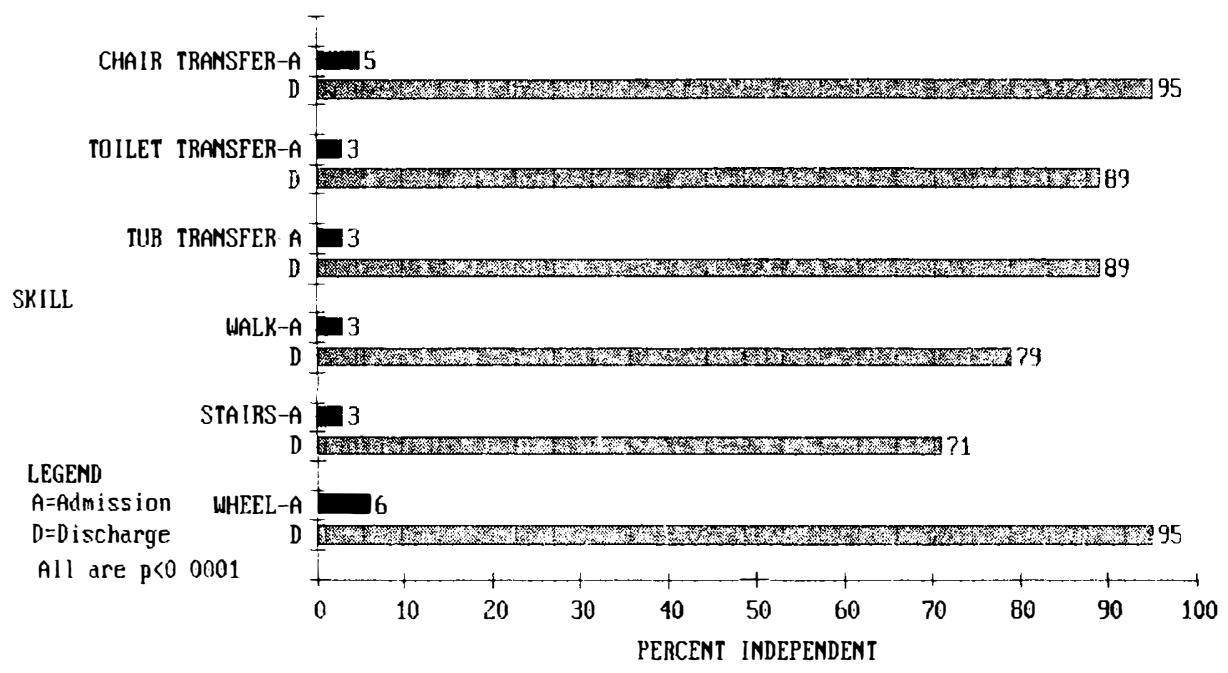

Figure 3 Proportions of patients who were independent in 6 mobility skills.

the 9 outcome variables were statistically significant. Patients who were discharged home had greater mean educational levels than were those who were discharged to nursing homes $(t=7 \cdot 0 ; p<0 \cdot 0001)$. Patients whose injuries resulted from road traffic accidents were more likely to be employed at follow-up than were those who were injured by other means $\left(X^{2}=3.91 ; p<0.05\right)$. Patients who underwent surgical cervical spinal stabilisation tended to have shorter lengths of rehabilitation stay $(t=$ $2.5 ; p<0.05)$. The most important predictor of function was whether the upper or lower limb was the predominant site of weakness; when the upper limb was weaker than the lower limb, patients demonstrated a greater likelihood of walking at discharge $\left(\mathrm{X}^{2}=4.25 ; \mathrm{p}<0.05\right)$, greater discharge MBI mobility subscores $(\mathrm{t}=2.5$; $p<0.05)$, and greater discharge MBI total scores $(t=2.3 ; p<0.05)$. None of the other potential predictive factors was significantly associated with any of the other outcome measures.

\section{Discussion}

The purpose of this study was to conduct a comprehensive examination of patients with partial or complete trauma-induced cervical spinal hemiplegia. Prior authors have described clinical features of this syndrome, but of ten with a different focus from the one in this investigation (Taylor and Gleave, 1957; Bosch et al., 1971; Little and Halar, 1985; Cabezudo et al., 1980; Cushing, 1894; Gentleman and Harrington, 1984; Holmes, 1915; Lipschitz and Block, 1962; Peacock et al., 1977; Preston, 1896; Rand and Patterson, 1929; Koehler and Endtz, 1986).

In this report, the broad ranges of backgrounds and injury types indicate that this syndrome does not have a uniform presentation. The paucity of patients with stabwounds as a cause of partial or complete spinal hemisection is of note because this syndrome traditionally has been associated with penetrating injuries (Lipschitz and Block, 1962; Peacock et al., 1977). However, the relative rarity of stabwoundinduced SCI has been noted in the past (Cabezudo et al., 1980; Gentleman and 
Harrington, 1984; Koehler and Endtz, 1986; Rand and Patterson, 1929; Guttman, 1973). A variety of aetiologies, including those which result in closed spinal injuries with or without vertebral fractures, may cause BSS. As a consequence, the SCI may be associated with injuries in other organ systems.

Despite this variation in presentation, considerable consistency was found in the prognosis of BSS and BSPS. Sizeable proportions of patients made neurologic recovery (nearly all), improved functional abilities (all), ambulated independently at discharge (three-fourths), independently performed their functional skills at discharge (at least two-thirds), achieved bladder and bowel continence by discharge (nearly all), were discharged home (nearly all), and even regained employment (one-third).

The general expectation of neurological and functional recovery has been noted by other authors, who focused primarily on motor recovery (Bosch et al., 1971; Little and Halar, 1985; Taylor and Gleave, 1957). In this investigation, evaluation of motor function was supplemented by examination of sphincter control and of the ability to complete daily living skills. This is the first study to apply a standardised systematic functional assessment instrument to patients with BSS.

In the present study, only a small proportion of the sample (13\%) had the 'pure' or classical syndrome with complete hemiplegia and contralateral hemianesthesia. Some measures of outcome were found to be significantly less favourable for this group than for the BSPS group. This finding is in contrast to the assertion by Koehler and Endtz (1986), who stated that pure BSS might be expected to have a better prognosis than BSPS because both lower extremities are paretic in the latter condition.

While SCI has been described as 'one of the most devastating calamities of human life' (Guttmann, 1973), patients who have BSS or BSPS generally can be expected to have favourable neurologic and functional outcomes after rehabilitation. This study has demonstrated that all patients with these particular syndromes have the potential for excellent outcomes and for a return to near pre-injury lifestyles.

\section{References}

American Spinal Injury Association 1987 Standards for neurological classification of spinal injury patients. Chicago: American Spinal Injury Association

BOHLMAN HH 1979 Acute fractures and dislocations of the cervical spine-An analysis of three hundred hospitalized patients and review of the literature. F Bone foint Surg 61A:1119-1142.

BoSCH A, STAUFFER ES, NICKEL VL 1971 Incomplete traumatic quadriplegia-A ten-year review. F AmMed Assoc 216:473-478.

BraAKMAN R, PENNING L 1976 Injuries of the cervical spine. In: Vinken PJ, Bruyn GW(Eds.) Handbook of Clinical Neurology. Vol. 25 Injuries of the Spine and Spinal Cord, Part 1, pp. 227-380

BROWN-SEQUARDCE 1868 Lectures on the physiology and pathology of the central nervous system and on the treatment of organic nervous affections. The Lancet 2:593-595, 659-662, 755-757, 821-823

Cabezudo JM, Carrillo R, Areitio E, Garcia de Sola R, VaqueroJ 1980 Accidential stab wound of the cervical spinal cord from inf ront. Acta Neurochirg 53:175-180.

CusHING HW 1898 Haematomyelia from gunshot wounds of the spine. Amf Med Sci 115:654-683.

DUNDAS R 1825 Case of concussion of the spine, tending to confirm the opinion that the nerves of sensation and of motion are distinct. EdinburghMed f 23:304.

FraNkel H, HaNCOCK DO, Hyslop Get al. The value of postural reduction in initial management of closed injuries to the spine with paraplegia and tetraplegia. Paraplegia 7: 179-192.

GENTLEMAN D, HARRINGTON M 1984Penetrating injury of the spinal cord. Injury 16:7-8.

GRANGER CV, ALBRECHT GL, HAMIL TON BB 1979Outcome of comprehensive medical rehabilitation: Measurement by PULSES profile and the Barthel index. Arch Phys Med Rehabil 60:145-154. 
TRAUMATIC CERVICAL BROWN-SEQUARD SYNDROME 589

Guttmann L 1973 Spinal Cord Injuries. Blackwell Scientific Publications, Oxford.

HoLMES G 1915 The Goulstonian lectures on spinal injuries of warfare. BrMed 2:855-861.

KOEHLER PJ, ENDTZ LJ 1986 The Brown-Sequard syndrome-True or false? ArchNeurol 43:921-924.

LIPSCHITZ R, BLOCK J 1962 Stab wounds of the spinal cord. Lancet 1:169-172.

LITTLE JW, HALAR E 1985 Temporal course of motor recovery after Brown-Sequard spinal cord injuries. Paraplegia 23:39-46.

MAHONEY FI, BARTHEL DW 1965 Functional evaluation: The Barthel index. Maryland State Med 14 : 61-65.

PeAcock WJ, Shrosbree RD, Key AG 1977 A review of 450 stabwoundsof the spinal cord. $S$ Afr MedF 51:961-964.

PRESTONGJ 1896 Brown-Sequard paralysis with report of a case. F Nerv Ment Dis 21:645-651

RANDCW, PATTERSONGH 1929Stab wounds of the spinal cord-Report of seven cases. Surg Gynecol and Obstet 48:652-661.

TAYLOR RG, GLEAVE JRW 1957: Incomplete spinal cord injuries-with Brown-Sequard phenomena. F Bone foint Surg 39-B:438-450.

YARKONYGM, RoTh EJ, HEINEMANN AW, Wu YC, KATZ RT, LOVELL L 1987Benefitsof rehabilitation for traumatic spinal cord injury: Multivariate analysis in 711 patients. ArchNeurol 44: 93-96. 